\title{
Dynamiques Créatives d'un Patrimoine Immatériel: la transmission orale du théâtre dansé Bharata-Nâtyam à Mysore (Inde)
}

\author{
Katia Légeret \\ Université Paris 8 - Paris, France
}

RÉSUMÉ - Dynamiques Créatives d'un Patrimoine Immatériel: la transmission orale du théâtre dansé Bharata-Nâtyam à Mysore (Inde) - Les chercheurs qui s'intéressent aux patrimoines des théâtres dansés en Inde rencontrent un obstacle majeur, celui de leur oralité, car les archives écrites et audiovisuelles sont rarissimes. Ils doivent suivre une formation professionnelle pour avoir accès aux matériaux de recherche et pouvoir les interpréter. L'étude de cas du style Bharata-Nâtyam de Mysore, implique cette observation participante du chercheur-artiste et démontre que la vitalité de cet héritage tient à la créativité de chaque transmission locale et individuelle - selon son contexte social, esthétique, religieux et politique - et non aux œuvres dites achevées et reconnues par un public national ou international.

Mots-clés: Transculturalité. Patrimoine Immatériel. Théâtre et Danse. Théâtre de l'Inde. Bharata-Nâtyam.

ABSTRACT - Dynamism and creativity of an immaterial heritage: the oral transmission in Bharata-Natyam Dance Theater (Mysore, India) - Researchers interested in the heritage of dance-theater in India face a major obstacle: oral transmission, as written and audiovisual archives are extremely rare. They need professional training to access research materials and to interpret it. The case study of the Mysore Bharata-Natyam style involves this kind of participatory observations by the researcher-artist and demonstrates how the vitality of this heritage depends on the creativity of each local and individual transmission - related to its social, aesthetic, religious and political context - therefore, not on works considered complete and recognized by a national or international public.

Keywords: Transculturality. Immaterial Heritage. Theater and Dance. India Theater. Bharata-Nâtyam.

RESUMO - Dinâmicas Criativas de um Patrimônio Imaterial: a transmissáo oral do teatro-dança Bharata-Nâtyam em Mysore (Índia) - Os pesquisadores interessados pelos patrimônios do teatro-dança indiano encontram um grande obstáculo: o da oralidade, pois os arquivos escritos e audiovisuais são raríssimos. Os pesquisadores devem passar por uma formação profissional para ter acesso aos materiais de pesquisa e poder interpretá-los. $\mathrm{O}$ estudo de caso do estilo Bharata-Nâtyam de Mysore, envolve esta observaçáo participante do pesquisador-artista e mostra que a vitalidade desta herança depende da criatividade de cada transmissão local e individual - segundo seu contexto social, estético, religioso e político - e não das obras ditas acabadas e reconhecidas por um público nacional ou internacional.

Palavras-chave: Transculturalidade. Patrimônio Imaterial. Teatro e Dança. Teatro Indiano. Bharata-Nâtyam. 
En 1932, la caste des danseuses de temples est menacée de disparition par la colonisation anglaise. Depuis leur arrivée en Inde en 1858, les colons s'opposent en effet aux rituels dansés dans les sanctuaires hindous. Grâce au courant réformateur de la Music Academy et de Kalakshetra, institutions créées à cette occasion à Chennai, en Inde du Sud, cet art des temples appelé Sadir, est alors renommé Bharata-Nâtyam. Ce néologisme sanscrit signifie l'art dramatique (nâtya) de Bharata, qui est à la fois un héros légendaire et l'auteur du traité le plus ancien commun aux arts de la scène (Nâtya-Shâstra). Ce nouveau nom est donc volontairement destiné à rappeler le caractère ancestral de cette danse et sa continuité historique avec le théâtre, la musique, la langue sanscrite et les textes sacrés védiques. Ce gage d'authenticité et de respectabilité ne doit pas cependant occulter un certain nombre de transformations subies par cet art en Inde du Sud. Les plus importantes pourraient être résumées en trois points. Pour se produire, les artistes passent du temple - ou bien du palais et de la maison - à une scène de type occidental proposée par les deux académies que nous venons de citer et accessible à un public international. Cet art se démocratise, il est pratiqué par d'autres castes, par des non-hindous et par des étrangers. Enfin, sous la pression de la pudibonderie victorienne, les textes et les gestes trop explicitement érotiques ont été écartés des répertoires.

Actuellement, les écoles de Bharata-Nâtyam se comptent par centaines, elles sont présentes dans les grandes villes de l'Inde et c'est le style le plus pratiqué et le plus programmé à l'étranger. Mais que reste-t-il encore de ce qui a été transmis depuis les années trente? Peut-on parler de continuité historique de génération en génération? Cet art étant fondé sur une transmission exclusivement orale, est-il possible de le préserver en tant que patrimoine? Certes, la patrimonialisation est visible dans les grandes écoles, qui enseignent des répertoires établis et pratiquement identiques pour tous les élèves. Or, d'autres formes d'identités culturelles et artistiques, notamment à Mysore, se distinguent fortement des transformations imposées par l'État du Tamil Nadu en 1932 avec la re-naissance du BharataNâtyam ${ }^{1}$, qui privilégie notamment la langue officielle (le tamoul) et interdit les mouvements acrobatiques dansés. Le style de Mysore est en effet caractérisé par des compositions musicales liées à la tradition des danseuses de cour et aux poètes de langue kannada. Mais plus encore, il se définit par l'importance extrême donnée au 
jeu corporel de l'acteur, et la très grande souplesse requise pour cette fonction théâtrale lui permet d'interpréter des personnages spécifiques, tels des animaux ou des éléments de la nature et d'enrichir le texte narré par la richesse de son vocabulaire gestuel. Il m'a été donné de constater depuis trente ans, que le caractère identitaire de cet héritage du style de Mysore tient à la vitalité du processus de création et non aux œuvres elles-mêmes. Autrement dit, la garantie de sa conservation patrimoniale pourrait-elle se mesurer, d'une part à sa négation de toute composition achevée, et d'autre part au dynamisme de sa transmission en Inde mais aussi à l'étranger? Dans ce cas, une recherche purement théorique et de type académique sur la question, s'intéressant à l'analyse des spectacles programmés dans les théâtres, risquerait de conclure à des répertoires certes variés, mais figés dans leurs codifications.

Le projet de préserver des répertoires anciens serait donc indissociable du processus vivant de leur transmission - tant sur le plan local qu'international - et de leur recréation, loin de toute répétition ou reconstitution. C'est pourquoi la définition de ce genre de patrimoine n'est pas réductible à un recensement d'œuvres et d'écrits. Car ce sont les artistes eux-mêmes qui les font vivre, en étant capables de les transformer, selon un certain nombre de règles. Cependant, les artistes indiens sont formés en grande majorité par un seul professeur, ils restent fidèles à leur style et à ma connaissance, ils ne s'intéressent pas à l'étude comparative des répertoires. Cette démarche consistant à varier les sources caractérise au contraire l'artiste-chercheur. S'il s'intéresse à cette question de patrimoine, il doit avoir un accès direct aux processus de transmission orale (in situ ou enregistrés) et, à partir de son savoir-faire et de son savoir-expert, il peut traduire les langages corporels codifiés. Cette situation privilégiée appartient aux étrangers ayant reçu une formation en Inde. En effet, ce statut hors caste leur permet de suivre plusieurs enseignements, de rencontrer de nombreux artistes et d'avoir une expérience assez riche pour envisager des recherches universitaires en arts de la scène de ce type lorsqu'ils sont en master et surtout en doctorat. Elles sont encore très rares. Il nous a été donné de recevoir en trente ans plusieurs formations de Bharata-Nâtyam, en France avec Amala Devi, puis en Inde avec K.Swarnamukhi, Venkatalakshama et K. Muralidhar Rao.

Nous analyserons des exemples précis de transmission du Bharata-Nâtyam, par les deux personnalités les plus marquantes de cet 
art à Mysore depuis une cinquantaine d'années: Venkatalakshama et K. Muralidhar Rao. Plusieurs questions ont sous-tendu le protocole de notre méthode d'investigation. Un même texte de répertoire est-il composé par le maître de multiples manières? Si tel est le cas, dépendt-il des musiciens qui l'entourent? De son lieu de transmission? De l'origine sociale de l'élève? De l'érudition du vocabulaire gestuel? De la diversité des formations artistiques? Notre apprentissage d'une quarantaine de répertoires nous a permis de partir du postulat suivant: plus les variations d'interprétation sont riches, plus la préservation du répertoire pourra être assurée; mais à l'opposé, la simplification de ses formes exigée par un certain type de construction identitaire, met en péril les principes de sa créativité et l'arrêt, souvent brutal, de sa transmission. Déceler et recenser ces différences selon les degrés d'intensité de la créativité, implique d'être un chercheur-artiste, c'està-dire ayant un niveau professionnel dans le style étudié.

\section{Le Postulat d'une Esthétique de la Variation}

L'enjeu est de montrer comment la constitution de ce patrimoine est un processus vivant, instable et fragile. Il se fonde sur une idée très particulière de la continuité historique, structurée par plusieurs éléments, qui feront l'objet des trois parties de cette étude. Notons avant de commencer les deux plus importants: une certaine idée esthétique de la variation, propre à chaque répertoire et la vitalité des communautés d'artistes hors d'un cadre institutionnel. Dans ce milieu artistique de Mysore, une mise en scène est dite belle lorsqu'elle est vicitra, ce mot sanscrit signifiant ce qui est orné, bariolé, varié. En effet, la continuité entre les répertoires passés et présents dépend de la créativité orale du transmetteur, c'est-à-dire de l'invention constante de variantes, réalisées avec des éléments nouveaux et étrangers. La difficulté pour comprendre ce processus tient au fait que ces variations s'élaborent avec une connaissance littéraire érudite des différentes interprétations d'un même épisode mythique. Le maître de Bharata-Nâtyam les met en scène, avec une série de personnages que son disciple soliste devra jouer à lui seul, passant sans cesse d'un rôle à un autre. En outre, chacun des mots racontant cet épisode est orné par un langage non verbal très élaboré. Par exemple, il existe des micromouvements rythmiques de l'acteur-danseur, appelés recaka. Ils sont faits de minuscules mouvements - rotatoires et dans plu- 
sieurs directions - des épaules, du cou, des poignets ou des sourcils. Ils viennent perturber la narration, la ralentir ou l'accélérer, jusqu'à créer parfois des contretemps et des sous-rythmes. Cette technique corporelle est mise en valeur par les percussions de l'orchestre, par les modulations de la voix du chanteur et par celles des instruments mélodiques comme la flûte.

Dans le langage musical carnatique, propre à l'Inde du Sud, les recaka correspondent au mot gamaka signifiant qu'une note est soudain ornée d'une succession d'oscillations, de glissements ou de mordants. De même que pour le danseur un recaka du poignet lui permet de passer subtilement d'une position de main à une autre, le gamaka crée, pour le musicien, un passage progressif mais très rapide d'un quart de ton entre deux notes. C'est justement cette sorte de reconstruction orale, corporelle, savante, technique et quasi invisible d'un répertoire du passé, qui va articuler d'une manière nouvelle les mots, les gestes et les sons. Cette créativité, liant de manière intrinsèque la poésie, la danse, le théâtre et la musique, modifie donc sans cesse la signification historique originale du texte mis en scène. Or, analyser ce processus implique, pour le chercheur-artiste que nous sommes - recevant l'enseignement de K. Muralidhar Rao depuis 1985 - la méthode d'une observation participante sur le terrain à Mysore, dont peu d'étrangers bénéficient.

Voici un dernier élément important à prendre en compte dans cette réflexion. Contrairement au Tamil Nadu qui a institué le Bharata-Nâtyam dans la ville de Chennai et à partir d'une rupture radicale avec les castes et les temples, le patrimoine de Mysore, lui, est constitué par la diversité renouvelée des lieux de formation et de création, en relation constante avec les temples et avec le palais du maharaja Ce Bharata-Nâtyam est en grande partie un art de cour, fondé sur un jeu d'acteur (nâtya) considéré comme étant supérieur à la danse pure (nritta) et il donne une place majeure aux musiciens de langue kannada. C'est pourquoi les maîtres qui transmettent le Bharata-Nâtyam à Mysore depuis plus d'un demi-siècle, tel K. Muralidhar Rao, ne se reconnaissent pas dans la traduction usuelle en anglais du terme Bharata-Nâtyam telle qu'elle a été diffusée dès 1932 par Rukmini Devi, directrice de l'institution Kalakshetra et par son mari anglais, Dr. Arundale. En effet, l'idée de traduire cet art par Indian Classical Dance a été conseillée à Rukmini Devi par Anna Pavlova, célèbre danseuse étoile du Bolchoïrusse² ${ }^{2}$ Grâce à elle, 
Rukmini Devi se forma à la danse classique occidentale avant de commencer l'apprentissage du Bharata-Nâtyam à l'âge de trente ans, ce qui est exceptionnel pour une femme brahmane de son époque. Nous verrons, au contraire, comment le processus de patrimonialisation du Bharata-Nâtyam à Mysore dépend des communautés d'artistes locaux, réunissant des acteurs, des danseurs, des musiciens et des poètes.

\section{Privilégier les Multiples Variations du Code Gestuel pour In- terpréter un Texte}

Pour apprendre par cœur les cinquante et une positions de mains (hasta ou mudrâ), tous les artistes de Bharata-Nâtyam se réfèrent au même manuel écrit en sanscrit, l'Abhinayadarpana de Nandikeshvara, antérieur au $\mathrm{VI}^{\mathrm{e}}$ siècle. Chaque geste possède plusieurs significations, dont la liste est récitée en sanscrit pendant l'apprentissage de cet art et toujours dans le même ordre. Par exemple, sarpa-shîrsa, tête de serpente, comprend d'abord ces actions successives: appliquer la pâte de santal (candana), montrer un serpent (bhujana) (Image 1), parler à voix basse (mandra), consacrer quelque chose en l'aspergeant d'eau (proksana) et choyer un être (posana-âdi). Certes, à un certain niveau, nous pouvons considérer que ce texte fait partie du patrimoine du Bharata-Nâtyam. Toutefois, il n'aide pas à élaborer un répertoire de mises en scène et de chorégraphies ni à caractériser le processus de patrimonialisation de ce style à Mysore. Car il n'y a pas de collage systématique, fixe et stable entre chaque mot d'un texte que l'acteur doit jouer et un geste précis et unique qui lui correspondrait. Il a toujours le choix entre plusieurs possibilités et il pourra aussi envisager de répéter plusieurs fois un mot et de l'exprimer avec des gestes différents, montrant ainsi la subtilité et la richesse de son interprétation du poème mis en scène. Cette liberté explique la diversité des styles de Bharata-Nâtyam. 


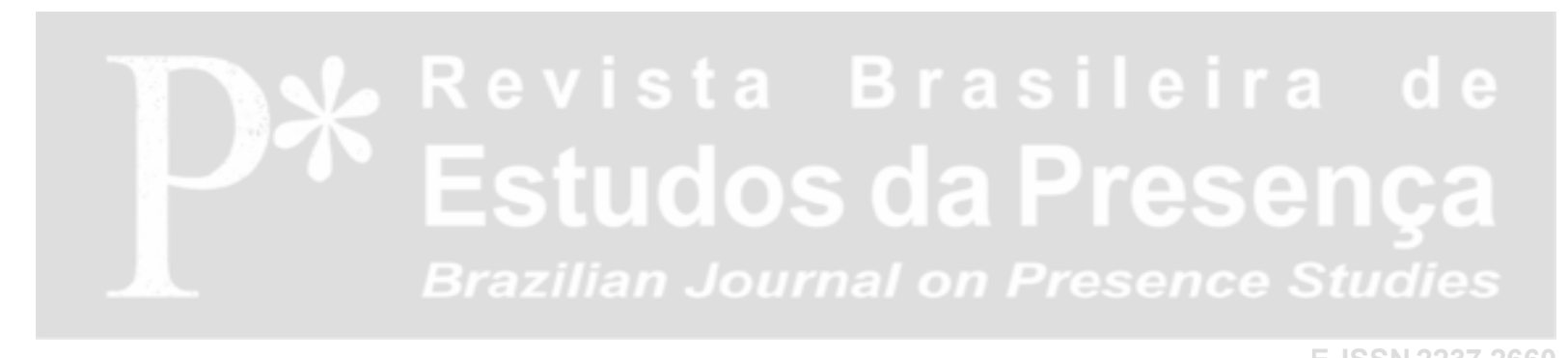

E-ISSN 2237-2660

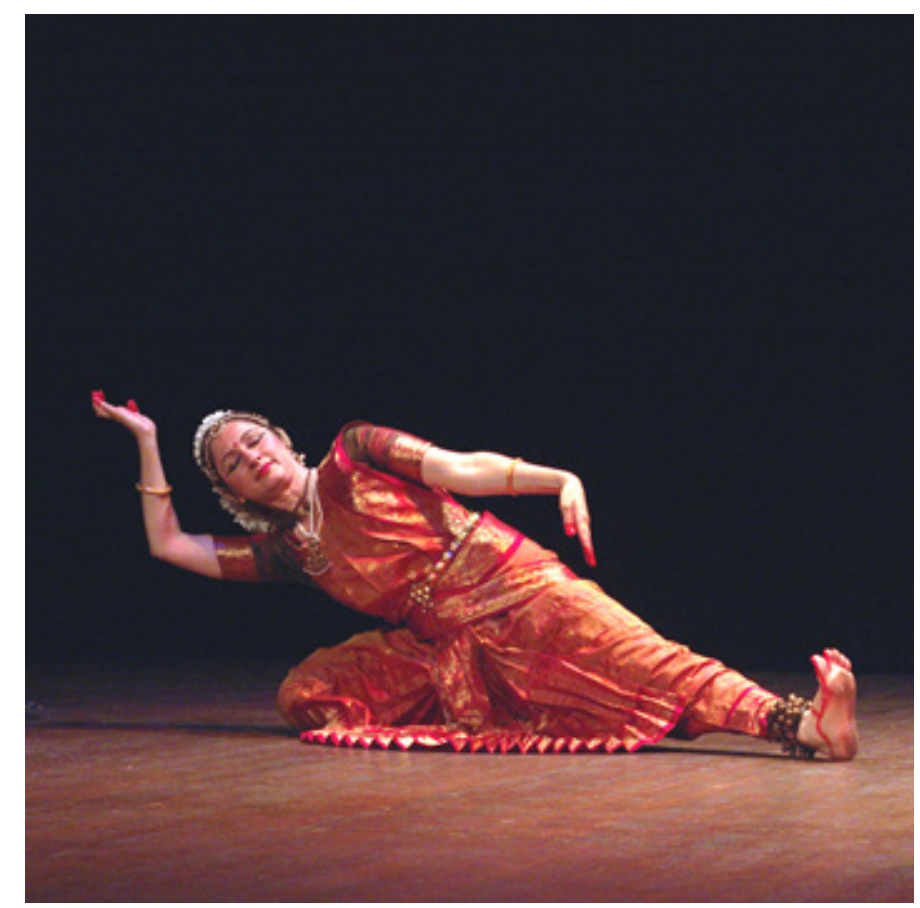

Image 1 - Kátia Légeret interprète le dieu Vishnu, couché sur le serpent cosmique Ananta (Manochhaya). Photo: Katia Légeret.

Prenons l'exemple de deux transmissions du même texte, que nous avons reçues à Mysore en 1985 et 1986 par Venkatalakshamma, la dernière danseuse de cour puis par K. Muralidhar Rao, le maître le plus important à Mysore depuis 1965. Lorsque la Gita-Govinda de Jayadeva, poème sanscrit du XII e siècle, décrit l'hérö̈ne Radha attendant le dieu Krishna, il serait simpliste d'associer le geste le plus illustratif à chaque mot de ce vers et par conséquent de le traduire littéralement avec quatre positions de mains: elle regarde (paçyati) de tous côtés (diçi diçi) et en secret (rahasi) votre Seigneur (bhavantan). Cela reviendrait à manier une sorte de langue des signes, visant à communiquer efficacement et non à suggérer poétiquement. Venkatalakshamma, elle, enseigne et chante dans la maison d'une de ses élèves. Comme elle le fait aussi à la cour du palais, elle reste assise en tailleur sur une natte pendant que son élève se déplace avec quelques pas très simples et sur une surface très réduite.

Dans cet espace intimiste et pour interpréter ce vers, toute l'attention du professeur va être concentrée sur les variations de l'état émotionnel suggérées dans le passage d'un mot à un autre. Pour ce faire, une seule mudrâ est choisie, sarpa-shîrsa, que nous venons de citer plus haut. Or, au lieu de se limiter aux huit sens donnés par 
l'Abhinayadarpana, cette artiste invente cinq autres significations tout en gardant toujours cette position des doigts serrés avec les deux dernières phalanges légèrement incurvées vers l'avant - suggérant ainsi une tête de cobra comme l'indique son nom. Avant de regarder autour d'elle, l'héroïne Radha masque son visage avec ses deux mains et lève très lentement le regard: est-elle triste d'être seule? Vient-elle de pleurer? Lorsqu'elle tourne la tête de tous côtés et place ses mains devant sa bouche, est-elle surprise en entendant un bruit? A-t-elle peur? Et dans ce dernier cas, sa tête reste immobile, seuls ses yeux vont et viennent de droite à gauche pendant que le haut de son dos se voûte légèrement, ses épaules s'affaissent et elle se fait toute petite. Ces actions ayant lieu en secret, ses mains viennent presser sa poitrine et son regard suggère un sentiment amoureux. Puis, soit elle invoque son Seigneur en le montrant devant elle avec ses deux mains qui s'élèvent lentement, suggérant sa grandeur, soit elle dessine devant elle, dans l'espace vide, la forme de son corps, ou encore elle prend elle-même la pose d'un personnage masculin, en posant ses deux mains, toujours en sarpa-shirsa, sur ses cuisses.

La transmission et la mise en scène de ce même texte par le maître K. Muralidhar Rao est très différente. Il propose à ses acteursdanseurs une sorte d'exercice d'improvisation, qui constituera ensuite la matière de sa composition. En effet, il leur demande de décrire, avec leur langage gestuel, le contexte temporel, local et géographique du texte, qui vient enrichir l'expression de chaque mot. Pour en faciliter l'élaboration, cette même phrase sera répétée un grand nombre de fois par le chanteur et l'action de l'acteur-danseur se déroule librement, sans s'attacher au sens littéral des mots. Dans le cas précis que nous étudions, la question essentielle est ce que voit Radha autour d'elle: s'agit-il d'une maison ou d'un lieu extérieur? Quelles sont les formes et les bruits dans lesquels elle croit reconnaître l'arrivée de Krishna? L'artiste est conduit à développer un contexte réaliste, lié au lieu où elle vit et dont certains éléments seraient précisés ailleurs dans la Gita-Govinda. Mais il peut être aussi le fruit de son imagination, ou encore se fonder sur son vécu personnel d'une rencontre amoureuse. Par conséquent, il serait possible d'affirmer que ce quatrième ashtapati de la Gita-Govinda fait partie du patrimoine du Bharata-Nâtyam à Mysore, seulement si ses mises en scène témoignent du contexte social et culturel spécifique à cette ville, permettant aux artistes d'inventer des variations et d'enrichir les codes gestuels décrits dans l'Abhi- 
nayadarpana. Etant donné le nombre considérable d'interprétations différentes, une recherche exhaustive des formes de créativité dans cette même localité semble exclue. Et si tel était le cas, le chercheur devrait non seulement être formé à cet art mais franchir le seuil des maisons où cet enseignement a lieu, transgressant difficilement les frontières entre les familles et les castes d'artistes. Il resterait comme solution la captation cinématographique de ces danses lors de représentations publiques, bien que cette méthode occultât le processus de transmission, avec ses errances, ses improvisations et les commentaires in situ du maître.

\section{Une Mise en Scène Interculturelle entre les Gestes Dansés, les Syllabes Rythmiques et les Langages Poétiques}

Le fait d'introduire un texte dans les morceaux de danse hérités notamment du Tamil Nadu, l'état voisin du Karnataka, leur donne à la fois de nouvelles significations et une forme de continuité historique assimilable localement. C'est le cas de la première danse du répertoire élaboré au XIX siècle à la cour de Tanjore. Il s'agit de l'alarippu, comprenant un ensemble de mouvements dansés fondamentaux du Bharata-Nâtyam, articulés par des syllabes rythmiques, par exemple sur huit temps Tam ta dit tam dit tam dit tei ta ki ta ta $k a t e i$. Or, depuis les années quatre-vingt-dix, ce morceau est de plus en plus absent des récitals professionnels, certainement parce qu'il fait partie des longues années d'apprentissage et du premier répertoire d'école, dans lequel sa chorégraphie est souvent simplifiée (Gaston, 1996, p. 270). K. Muralidhar Rao a eu l'idée d'introduire l'abhinaya ou "jeu d'acteur" dans ces mouvements abstraits. Il a intégré cet héritage du Tamil Nadu dans le patrimoine littéraire de Mysore en associant les syllabes rythmiques (sholukattu) et le poème chanté en sanscrit pour les cérémonies du temple principal de Mysore, dédié à la déesse guerrière Chamundeshwari ${ }^{3}$. Ce poème ou stotram, intitulé Mahishasura Mardini, a été écrit au VIII siècle par le maître spirituel Adi Shankaracharya. Les élèves de K. Muralidhar Rao le dansent au moins une fois par an au festival de Dasara, à l'entrée du Palais. Le dixième jour de cette manifestation, qui comprend pendant toute sa durée un grand nombre de récitals et de spectacles, le maharaja préside une procession à dos d'éléphant à travers la ville avec la statue de cette déesse, trônant également sur un éléphant ${ }^{4}$. 


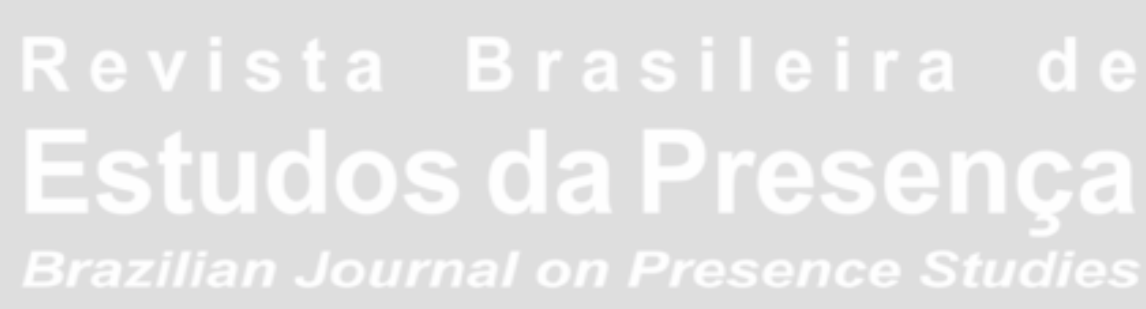

Détaillons un passage précis de ce texte dansé qui énumère une liste impressionnante des différents noms de cette divinité. Au moment où les syllabes rythmiques sont récitées à la troisième vitesse et au lieu d'accélérer ses déplacements, le danseur ralentit ses gestes et marque quelques poses sculpturales en s'immobilisant sur le cinquième temps de la phrase. Par exemple, il brandit un bâton, suggéré par deux points fermés placés sur une même diagonale, avec une double expression héroïque et colérique. De ce fait, les mouvements de l'alarippu, décrivant à l'origine des figures géométriques et purement rythmiques (adavu), se mettent à signifier un attribut de ce personnage. Ainsi, la position de main appelée kartari - la paire de ciseaux (Image 2) - avec l'index et le majeur qui se croisent, devient-elle une arme divine et redoutable. Justement, pendant ce dixième jour du festival, il est de tradition que tout ce qui est une arme soit béni. Or, il s'agissait autrefois de simples couteaux de cuisine et d'objets tranchants servant au quotidien dans une maison. Mais actuellement, les habitants de Mysore sélectionnent tout ce qui aide à réussir dans la vie, donc à vaincre les obstacles et les problèmes. Il est courant de voir des guirlandes de fleurs orner un Mobylette, un ordinateur ou les cymbales du professeur de danse, puisque ces objets sont indispensables à la survie de ceux qui les possèdent.

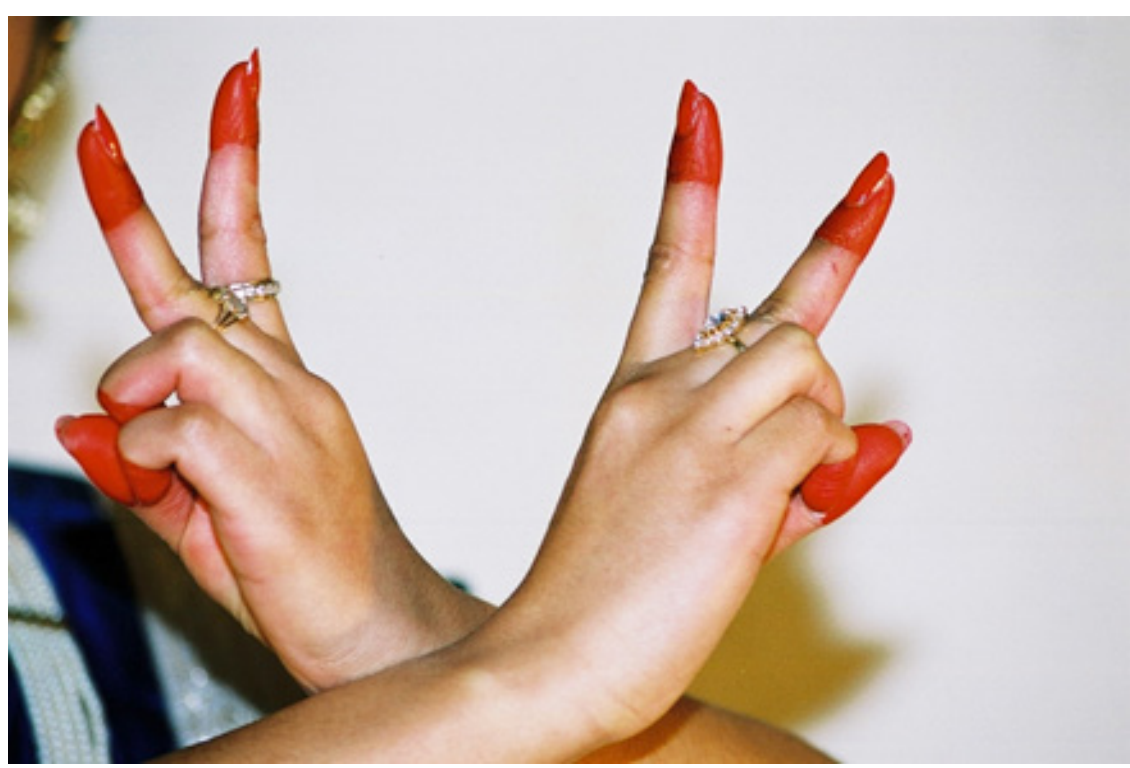

Image 2 - Les mains en kartari hasta. Photo: Katia Légeret.

Si nous nous demandons quelle est la continuité du répertoire de cour et de temple sur la scène contemporaine de Mysore, nous réalisons donc très rapidement qu'elle implique la présence implicite 
de langues plurielles. La patrimonialisation présuppose la traduction d'une langue dans une autre sans éliminer l'étranger. Au contraire, elle le préserve, comme nous l'avons vu dans cet alarippu qui juxtapose deux textes, celui des syllabes rythmiques et le poème à la déesse. Le théoricien indien postcolonial Homi K. Bhabha explique, dans Les lieux de la culture (Bhabha, 1994, p. 226) comment la traduction, lorsqu'elle est performative - donc apparaît en tant que récit oral met en scène la différence culturelle. C'est l'énonciation en acte, selon un mouvement permanent d'intertextualité, qui crée l'idée de continuité de l'histoire. Un texte est ainsi sous-tendu ou incrusté de manière fragmentaire par d'autres récits: ils s'absorbent et se transforment mutuellement. Nous nous sommes intéressés à appliquer cette théorie de la littérature aux arts de la scène et spécifiquement aux langages corporels. La composition des répertoires de Bharata-Nâtyam exprime cette idée de Homi K. Bhabha en considérant, depuis le XIX ${ }^{\mathrm{e}}$ siècle - selon l'état de nos connaissances actuelles - que le jeu d'acteur (sattvikabhinaya) est supérieur à la danse telle qu'elle a été imposée par les quatre célèbres compositeurs à la cour de Tanjores. Mais lorsque le maharaja de Mysore, Chamaraja Wadeyar invite à sa cour, entre 1881 et 1894, l'un des frères Cinnaya Pillai, celui-ci compose en majeure partie des morceaux d'abhinaya mettant donc en valeur l'aspect théâtral du Bharata-Nâtyam ${ }^{6}$. Le répertoire que Venkatalakshamma nous a transmis en 1985 lui vient de la danseuse de cour Jetti Thayamma, contemporaine de Cinnaya Pillai. Les compositions de Tanjore qu'elle a apprises étaient justement encadrées pendant plus de trois heures de récital par deux parties propres au style de Mysore ${ }^{7}$. La première, interprétée par l'artiste en chantant et en dansant - parfois assise en tailleur - comprend successivement un shurnikai, poème sanscrit dédié au roi ou à la divinité protectrice du récital ${ }^{8}$ puis un pushpanjali, offrande de fleurs. Vient ensuite une danse dédiée au dieu à tête d'éléphant Ganesh (ganapati stotram), suivie d'un shabdam racontant des épisodes de la vie du dieu Krishna (Légeret, 2005, p. 175-187). Sans rupture avec cette tradition, la dernière partie d'un récital actuel se termine en général par des pièces très jouées, narratives, en langue kannada?

Arrêtons-nous un instant sur le cas du pushpanjali. Avant de réapparaître sur scène dans les années 1970 comme une pièce purement rythmique, cette danse a été supprimée au XIX ${ }^{\mathrm{e}}$ siècle à la cour de Tanjore, puis en 1932 à Chennai pour rompre avec les rituels 
quotidiens des temples, qui considéraient cette offrande de fleurs comme essentielle. Les sculptures des sanctuaires témoignent de cette tradition, comme à Chidambaram ou à Tanjore, où l'on peut voir notamment le premier des cent huit karana, appelé talapushpaputa. Il représente l'entrée en scène de l'artiste, les mains chargées de pétales de roses, qu'il va offrir aux pieds de la divinité 5 Fig. $3^{\circ}$. Dans le Tamil Nadu, sous l'influence de maîtres tel K. Ellapa, le pushpanjali réapparaît sur scène dans les années 1970 comme une pièce purement rythmique. Les artistes commencent à placer en avant-scène une statue du dieu Shiva sous sa forme de danseur cosmique (Nâtarâja) et pendant ce morceau de répertoire, viennent lui déposer des pétales de fleurs (Gaston, 1996, p. 323). Or, l'art de Mysore n'a pas connu cette scission entre l'art de temple et l'art de cour. De surcroît, il n'a pas cessé de tisser les syllabes rythmiques de cette danse avec une trame narrative. L'une des compositions de K. Muralidhar Rao ponctue ainsi les passages de danse pure par des postures représentant le dieu Shiva et elle a fait partie entre 1970 et 1980 de certains rituels du célèbre temple shivaïte de Nanjangud près de Mysore. L'inspiration de ce maitre s'est fondée sur le témoignage de la famille de son élève Vani Doreswamy - chez qui il vivait alors - originaire de Nanjangud.

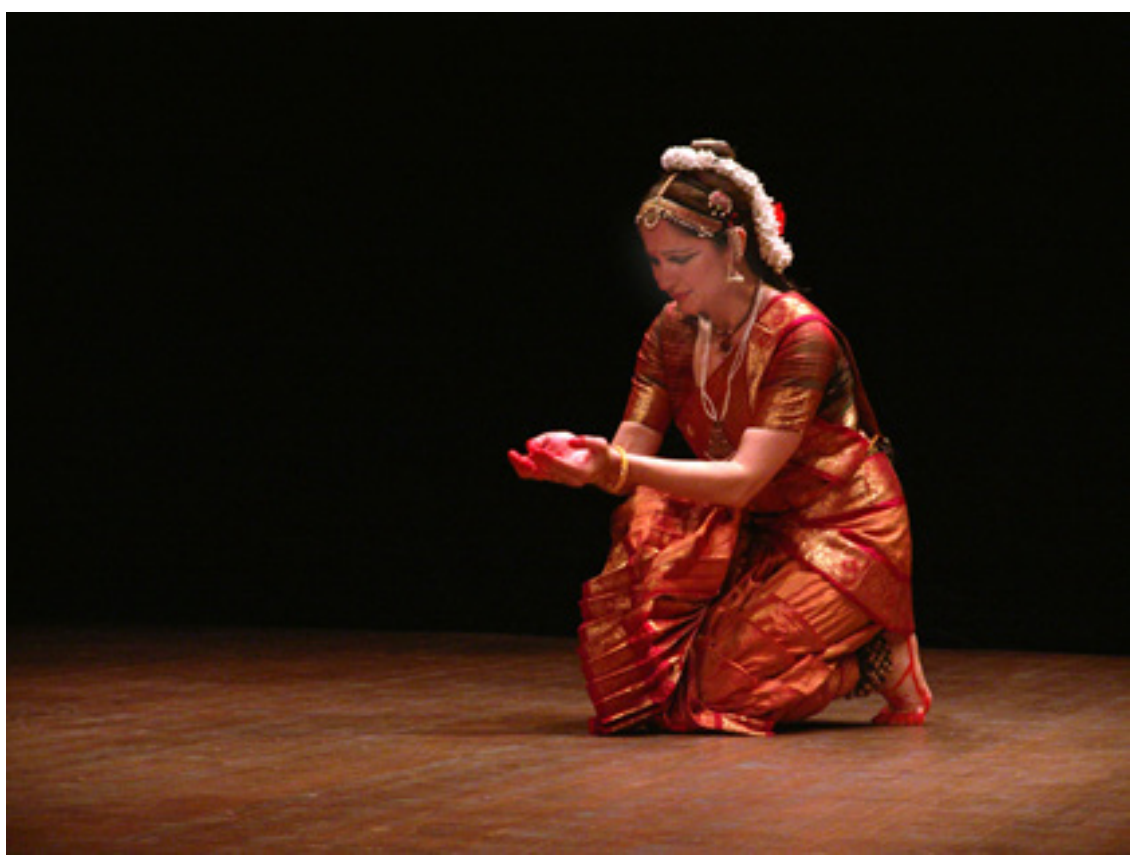

Image 3 - L’offrande de fleurs pushpanjali (Manochhaya). Photo: Katia Légeret. 
Elle nous rapporte que les danseuses de ce temple avaient alors trois répertoires, le premier ayant lieu dans le temple, le second appelé bhagavata mela se faisant à l'extérieur et le troisième s'accomplissant à la cour de Mysore. Ressemblant au pushpanjali tel que nous le connaissons encore actuellement, le bhagavata mela était effectué par cinq jeunes filles vierges et cinq femmes mariées, avec des parcours circulaires, la règle étant de ne jamais tourner le dos au public ni à la divinité. Après un ninayaka stotram, poème psalmodié pendant lequel elles se mettaient à plat ventre sur le sol, tenaient leurs chevilles en étirant les jambes vers le ciel, puis tournaient ainsi sur elles-mêmes. Avec cette figure extrêmement acrobatique, elles donnaient l'impression d'une roue mobile se déplaçant en cercle. Tout à la fin, elles s'allongeaient complètement sur le dos et restaient plusieurs minutes dans cette position pour évoquer le repos de la divinité.

Le récital actuel de Bharata-Nâtyam étant de nos jours principalement un art soliste, K. Muralidhar Rao a conservé le caractère circulaire des déplacements et une très grande variété et vélocité des pirouettes de l'artiste pour changer de direction et de personnage à interpréter. C'est ainsi qu'en 1986, K. Muralidhar Rao compose l'un des pushpanjali les plus connus dans le raga kedara, dont la phrase rythmique fondée sur huit temps est la suivante: Ta tom ta ta tom ta kountari kitatataka dit dit tei ta ka dit dit tei. Tous les quatre temps, l'artiste change de direction dans l'espace et montre les attributs symboliques d'une des neuf planètes. Il nous a également transmis sur cette même structure le shurnikai adyashri sashikananda: le texte sanscrit décrit le plaisir des trois plus grands dieux et de leur parèdre devant la danse du pushpanjali. Comme il est également adressé à Indra en tant que maître des directions, K. Muralidhar Rao élabore avec ses musiciens une composition - avec les percussions du midamgam, la flûte et la vina - sur laquelle l'acteur-danseur interprète chacune des dix directions de l'espace (dikpalaka) selon les attributs et la posture d'une divinité particulière, et selon une structure rythmique spécifique (Satyanarayana, 1966, p. 98-105). Notons en conclusion de ce chapitre qu'en général, le pushpanjali fait partie du premier spectacle public d'un danseur de Bharata-Nâtyam. Appelé en langue tamoule arangetram, il donne lieu à une grande cérémonie devant un grand nombre de personnes, événement extrêmement onéreux pour la famille. Mais à Mysore, Venkatalakshmanna, K. Muralidhar Rao et d'autres professeurs cultivent ce rituel tout à fait autrement. Sous 
le nom sanscrit de ranga pravesa, devant un public très restreint, l'élève accomplit les actions suivantes: il tient ses clochettes et récite le pushpanjali que nous venons de citer. Il salue ensuite les invités et les musiciens, avant de remettre ses grelots de cheville à son maître pour qu'il les bénisse. Puis les instruments de musique reçoivent la bénédiction du maître. Chacun jette des grains de riz à l'artiste qui se prépare à monter sur scène.

\section{Créer in Situ?}

Si les répertoires de Bharata-Nâtyam sont sans cesse remodelés et réagencés selon la vie communautaire du lieu où ils sont transmis, c'est avec une règle bien particulière: il n'y a pas de séparation entre les faits artistiques - relevant de l'action dans un but culturel précis et nouveau - et les faits anciens, qui impliquent une réflexion constante sur la fidélité à des règles précises de composition. Autrement dit, la créativité est le résultat d'une interprétation renouvelée des formes connues. Or, ce n'est pas ce que Gilles Tarabout, chercheur au Centre d'étude de l'Inde et de l'Asie du Sud constate, lorsqu'il analyse l'adaptation d'un rituel de peintures éphémères du Kerala, les kalams, sur une scène occidentale (Tarabout, 2003, p. 37-61). Il souligne au contraire l'écart absolu entre deux sociétés. D'un côté les programmateurs occidentaux argumentent sur le lien de cet art indien à un passé immuable, lointain et à un monde divin. De l'autre côté, lorsque les indiens dessinent sur le sol, ils pensent leurs actions en terme d'efficacité, ce processus esthétique étant éphémère, «[...] une beauté active, support efficient des divinités et hommage charmeur qui leur est adressé, au cours de rites dont larpeintures ne constitue qu'une séquence, essentielle et temporaire» (Tarabout, 2003, p. 43). Or, lorsque le dessin du kalam devient une sorte d'installation-performance dans un festival français, sa transformation est radicale:

L'histoire du possible passage des kalam à l'art est donc celle d'une décontextualisation fragmentéesde leur réalisation (fragmentée en ce sens que les artistes)continuent à être des officiants en dehors des expositions), et de leur recontextualisation selon les catégories occidentales de l'art et de la culture, où ils acquièrent des valeurs différentes (Tarabout, 2003, p. 43).

Reprenons l'exemple du Bharata-Nâtyam de Mysore, ce que Gilles Tarabout pose comme contradictoire ne l'est pas pour ces In- 
diens du Karnataka. Car leur créativité est générée par la scène où ils se produisent in situ, que ce soit en Inde ou à l'étranger. La question est de savoir comment cette adaptation fabrique de la nouveauté au sein des règles qu'ils se sont fixées a priori. Nous avons vu, lors de notre première partie, dans quelle mesure une même histoire pouvait se détacher du texte, se moduler, être détournée, se dérouler en quelques instants ou en une vingtaine de minutes. Ce sont les circonstances extérieures qui génèrent ainsi ces sortes d'improvisations. Par exemple, lorsque K. Muralidhar Rao parcourt l'Europe en 1988 pour une tournée de deux mois, son répertoire des Dashavatars, Les Dix Incarnations du Dieu Vishnu, s'adapte aux thématiques des lieux: «La créativité consiste à répondre à toute situation nouvelle dans la vie, avec la grammaire du Bharata Natyam et de constantes observation, analyse et contemplation de la nature» (Narayana Rao, 1999, p. 46).

Á Bergerac et à Périgueux, à l'occasion d'un festival Inde-Tibet, il me demandera, ainsi qu'aux quatre musiciens, de développer pendant une demi-heure au lieu de six minutes l'avant-dernière mise en scène dansée, consacrée à Bouddha, en tant que neuvième avatar de Vishnu. En juin 2001, invités au Festival des Musiques Sacrées de Fès (Maroc) dans le colloque Une Âme pour la Mondialisation, c'est Kalki (Image 4), le dernier des Dashavatars, qui est mis en valeur ${ }^{10}$. K. Muralidhar Rao choisit de traduire l'idée de comète $(k e t u)$ destinée à détruire l'humanité pendant cette époque de Kalki fléau qui s'abattra un jour sur l'bumanité par danger nucléaire et il met en scène un corps irradié, souffrant et mutant.

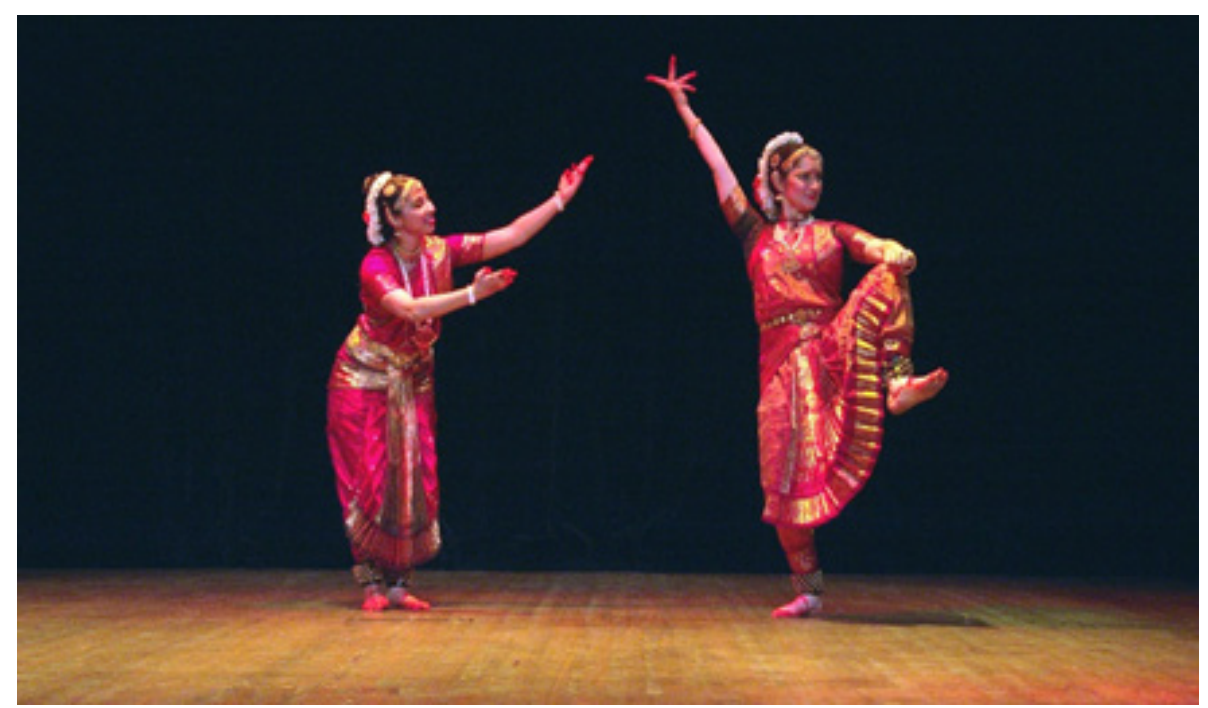

Image 4 - Kalki brandissant le feu (Manochhaya) et son dévot (Kaleyvani). Photo: Katia Légeret.

Katia Légeret - Dynamiques Créatives d'un Patrimoine Immatériel: 
Il est intéressant de rappeler qu'à Mysore, les lieux officiels de production et de diffusion du Bharata-Nâtyam sont avant ceux du patrimoine royal, même si le maharaja actuel n'a plus qu'un pouvoir symbolique. Le gouvernement lui donne une place d'honneur chaque année, lors du célèbre Festival Dashara. Des dizaines de spectacles ont alors lieu chaque jour et le répertoire correspond chaque fois à une thématique particulière. Pendant le reste de l'année - excepté au mois d'août, considérés comme non auspicieux - de nombreux répertoires d'artistes locaux et étrangers sont présentés au Jagamohan Palace, lieu de vie de la famille du maharaja transformé depuis plusieurs décennies en auditorium. Dans ce lieu très populaire, l'entrée est restée gratuite. Mais la durée des représentations doit s'adapter à plusieurs types de circonstances. Par exemple en temps de mousson, l'horaire peut être déplacé. Il arrive que la dernière danse soit supprimée si les horaires des derniers bus locaux sont avancés ou annulés au dernier moment. Citons également l'université de Mysore, qui comprend un théâtre et un département consacré au Bharata-Nâtyam, créé en 1965 grâce au mécénat du maharaja. Le premier professeur titulaire fut Venkatalakhmanna, qui y enseigna pendant neuf ans. En 1973, alors âgée de soixante-sept ans, elle obtint son doctorat de littérature. Sa fille, Shakuntala, continue à Mysore - principalement chez elle - la transmission de son art.

Nous avons pu constater comment ce qui est montré comme un répertoire achevé sur scène n'est pas fixe mais fluctuant et éphémère. La patrimonialisation se constitue selon les lieux de transmission, avec toutes les adaptations et la créativité qu'ils impliquent. Dans les années quatre-vingt, Venkatalakshmanna continue à enseigner de manière privée et elle change sans cesse de lieu, selon ce que ses élèves ont à disposition. Il peut s'agir d'un garage comme d'une chambre exigüe (Rosemary, 1998, p. 43). Cette artiste renommée est loin d'être riche, d'autant qu'elle a dû vendre un bon nombre de ses bijoux offerts par le palais, pour marier son fils, sa petite fille et pour payer une taxe importante dans sa communauté tribale des Lamban. Ces drames de la vie quotidienne ont souvent inspiré les thèmes de son enseignement. Lorsqu'elle m'apprend notamment en août 1985 un passage de la Gita-Govinda (ashtapati nataare), décrivant la douloureuse séparation des amants Krishna et Radha, elle ne parle pas un mot d'anglais et ses flots de larmes interrompent souvent les cours. Je n'apprendrai l'histoire de sa vie que beaucoup plus tard. Mais ces 
états émotionnels ont conditionné le mimétisme qu'elle me demandait pour capter sans explication verbale le moindre de ses gestes. La tension qu'elle imposait alors à ses mouvements pour maîtriser son chagrin exacerbait le style minimaliste de ses expressions du visage et de ses positions de mains. Elle était toujours assise sur une natte, répétait sans relâche les mêmes phrases, m’obligeant à reproduire le rythme irrégulier de sa respiration et les lignes de tensions partant de la poitrine, des épaules ou du ventre pour se propager jusqu'au bout des doigts. C'est pourtant grâce à ces circonstances malheureuses que j'ai pu expérimenter le sens littéral du mot sanscrit mudrâ, l'action de sceller, autrement dit de maitriser le souffle en bloquant à un moment précis à la fois l'expiration (ou l'inspiration) et un geste de mains.

K. Muralidhar Rao a également enseigné dans un grand nombre de lieux. Nous en avons connu une douzaine avec lui, depuis 1985. Il y eut des salles de classes dans une école de musique, le bureau, la terrasse puis le garage du journaliste K. Venkata Rao. Mais il s'agit toujours principalement du salon ou de la pièce principale de la famille chez qui il vit. Sans aucune contrepartie financière, depuis plusieurs décennies, il forme au Bharata-Nâtyam pendant un certain nombre d'années la fille de la maison, et en échange reçoit le gîte et le couvert. Il n'y a aucun horaire fixe pour les enseignements. Nous pouvons attendre une grande partie de la journée ou bien être interrompus en plein cours par une visite impromptue, un repas, la répétition d'un musicien ou le vacarme de la rue.

En 1998, il s'intéresse à la pièce citée au début du chapitre quatre du Nâtya-Shâstra, Le Barattement du Nectar d'Immortalité (amritta manthana). En quelques mois, il va composer trois mises en scènes différentes, non seulement parce qu'il a trois élèves mais parce que les lieux de transmission changent. Dans ce cas, le patrimoine de ce répertoire de Bharata-Nâtyam spécifique à Mysore n'est pas constitué par le texte racontant ce mythe du barattement mais par l'addition de leurs trois mises en scène et leur processus de création in situ. En voici la synthèse, fondée sur mon témoignage en tant que spectatrice et actrice. Tout d'abord, pour son élève Nandini V. Rangan dont la famille est vishnouite, il met en scène le curnikai siri mahalaksmi (raga arabhi) en montrant la déesse Lakshmi émergeant de ce barattement. L'idée lui est venue parce que le cours avait lieu pendant que cette élève aidait sa mère à préparer le repas et ne cessait de faire des allers et retours à la cuisine. Sa mère fredonnait en même temps le texte 
du shurnikai, qu'elle connaissait déjà par cœur, rythmant ainsi l'un de ses gestes quotidiens, celui de baratter le lait, autrement dit de transformer la crème de lait en beurre. Dans les années 1990, Nandini V. Rangan a fondé aux États-Unis une école de Bharata-Nâtyam à l'université de San Diego. Depuis 2004, elle enseigne son style de Mysore à l'Université Amritta du Kerala, fondée par la sainte du même nom et à qui elle dédie toutes les danses de son répertoire ${ }^{11}$.

Quant à son autre élève indienne, Vani Doreswami ${ }^{12}$, d'une famille de tradition shivaïte, il lui composa un devaranama du poète Purandaradasa, appelée chandrasuda en langue kannada. Insistant dans ce mythe du barattement - sur l'action du serpent céleste Vasuki, qui, épuisé de servir de corde pour tenir le mont Mandara, se mit à vomir son venin, menaçant de détruire l'univers. Ce maître a choisi un seul vers du poème, répété douze fois de suite, pour mettre en scène cette action. Pour sauver la création, le dieu Shiva but tout ce poison. Dans la version de Nandini, c'est la tortue Kurma (Image 5), qui résout le problème, en stabilisant sur son dos l'axe du mont Mandara servant de pilon au barattement.

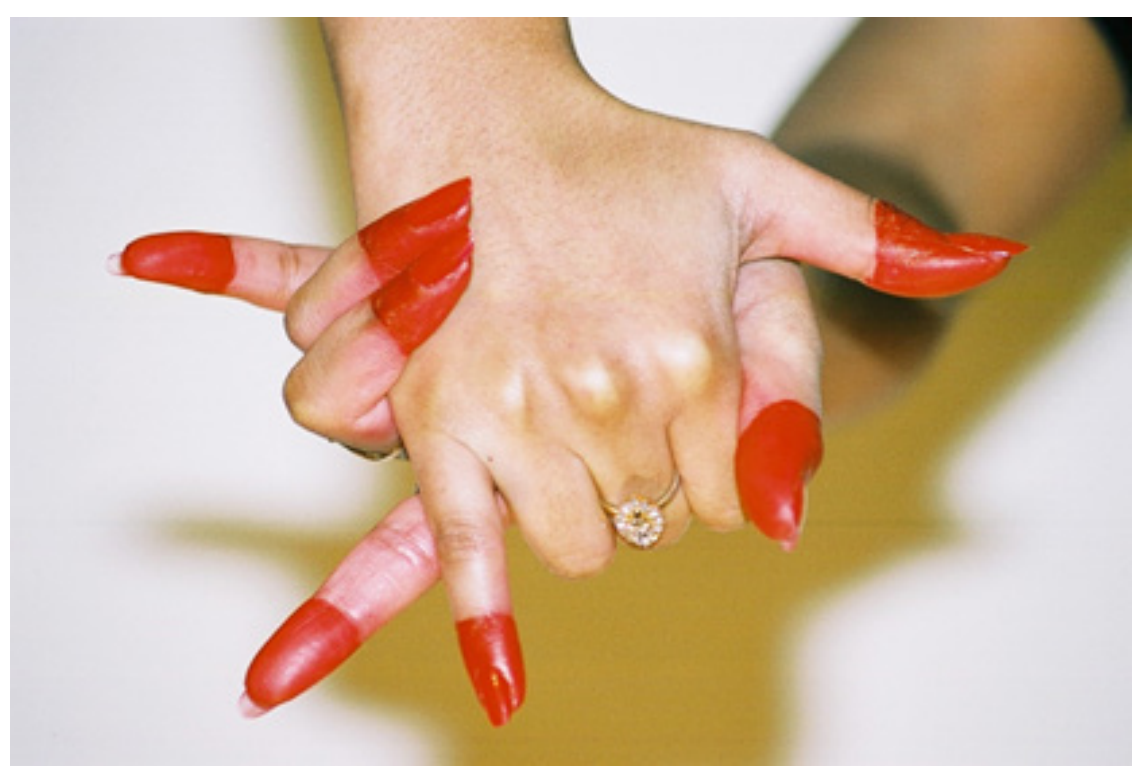

Image 5 - La tortue Kurma hasta. Photo: Katia Légeret.

La composition que K. Muralidhar Rao nous a transmise a tenu compte du public occidental à laquelle elle allait s'adresser et qui ne connaît pas ce mythe. Il créa une structure rythmique très élaborée de ce barattement, fondée sur le double mouvement opposé des dieux d'un côté et des démons de l'autre, tirant et poussant tour à tour la 
corde géante, jusqu'à impulser des mouvements rotatoires de plus en plus rapides au serpent et au mont. Tiré du premier poème de la Gita-Govinda, le vers est prononcé seize fois et sur une structure de seize temps. Accompagné par un duo de flûtes (l'une correspondant aux mouvements des dieux et l'autre à ceux du mont) et de percussions (le mridamgam interprétant les démons et les tablas le serpent), le chanteur joue sur la phonétique des mots qu'il décompose en syllabes rythmiques, effaçant leur sens littéral et narratif: kseti vati vipouratare tawa tishtati prischte / darani darana kina chakra garishte. Lors des tournées européennes en 1988 et 1989, les musiciens jouent un concert à de nombreux moments, dont cette partie du répertoire des Dashavatars. C'est pourquoi cette composition est intitulée sur les programmes: récital de Bharata-Nâtyam et non spectacle ou mise en scène.

Nous pouvons affirmer, pour conclure, que le patrimoine du Bharata-Nâtyam ne se définit ni par le nombre et la chronologie de ses répertoires, ni par ce qui lui appartiendrait ou ce qu'il aurait exclu. Mais il se caractérise par un mode spécifique de relation à l'étranger, qu'il s'agisse de textes, de langues, de professeurs, d'élèves ou de public. En effet, nous avons vu qu'il assimile et incorpore l'autre par un processus d'ornementation (vicitra), donc en le décomposant puis en le ré-agençant d'une manière nouvelle et originale. Une fable célèbre du Panchatantra, qui fait encore partie du répertoire des acteurs-danseur de Bharata-Nâtyam, intitulée Le Lion, le Corbeau, le Tigre, le Chacal et le Chameau illustre bien ce processus indien de patrimonialisation. Le personnage étranger incarné par le chameau, à qui le roi lion offre d'abord l'hospitalité, est finalement contraint de s'offrir en sacrifice, pour être dévoré par les animaux et leur permettre de survivre pendant une époque de famine. L'attitude opposée fait l'objet de la version que Jean de La Fontaine, notre fabuliste du XVII ${ }^{e}$ siècle, donne de cette même fable, dans Les Animaux Malades de la Peste. En effet, pour éradiquer l'épidémie, c'est l'âne qui est finalement exclu, représentant le rang social le plus bas de la société.

C'est pourquoi, l'idée de faire une étude exhaustive d'un tel processus de patrimonialisation, fondé à Mysore sur l'incorporation esthétique d'éléments étrangers, serait une tâche immense, dans la mesure où chaque transmission orale du Bharata-Nâtyam implique un genre spécifique d'assimilation. Citons dans ce sens et pour finir 
quelques exemples. Nous savons maintenant que Venkatalakshamma - décédée récemment - est considérée comme la dernière danseuse de cour et la plus célèbre de son temps. Elle a transmis son art à la caste lettrée et hindoue des brahmanes et aux enfants de ses collègues universitaires et littéraires. Or, son origine sociale est aux antipodes de ce milieu social. Elle est née en effet dans l'une des cinquante et une tribus du Kerala, hors caste et donc intouchable. Il s'agit des Banjara. Elle a appris avec eux la danse Kathak et des danses locales, liées aux rites de fertilité, ce dans un milieu d'agriculteurs semi-nomades venant du nord de l'Inde et parlant le lambadi, l'un des dialectes du Rajasthan. La danseuse Anupama, née à Mysore et formée par K. Muralidhar Rao depuis 1984, vit depuis plusieurs années à Francfort. Elle danse professionnellement le Bharata-Nâtyam mais elle fait aussi partie de la compagnie actuelle - fondée à Tours en France - des Dhoad, musiciens du Rajasthan. Car elle pratique leur art depuis son enfance et elle l'intègre dans son répertoire, avec des textes du Nord de l'Inde, en langues urdu et hindi. R. Keshavamurthy, artiste initié par Gundappa à la cour de Mysore, danse professionnellement le style kathak, qu'il a appris au Nord de l'Inde, avant de transmettre le Bharata-Nâtyam à Bangalore (Gaston, 1996, p. 168). K. Muralidhar Rao a grandi dans le district Kasaragod du Karnataka, là où vivent les plus grandes troupes de théâtre Yakshagana, qu'il a fréquenté pendant de nombreuses années. Il a été formé au style Kathakali, tradition spécifique à l'État du Kerala, avant d'enseigner le BharataNâtyam. Ces différentes influences étrangères sont reconnaissables dans sa transmission (Légeret, 2010, p. 71-73).

Dans tous les cas cités, où la vitalité du patrimoine tient à sa capacité créative d'assimiler ces éléments étrangers, il s'agit toujours de petits mouvements accomplis par différentes parties du corps. Ils apportent des variantes subtiles et un renouvellement du vocabulaire local et ils ouvrent ainsi ces textes anciens à de nouvelles transpositions, tout en impliquant des règles strictes et savantes. La connaissance des langages corporels codifiés en sanscrit, des langues véhiculant ces répertoires et de l'anglais qui fait partie de la transmission orale apparaît comme essentielle à ce type de recherche. Le chercheur-artiste contribue à la vitalité des processus de transmission car il donne en ce sens aux artistes des arguments transculturels qui dépassent leur intérêt local. En retour, le maintien d'une pratique artistique en 
Inde apporte au chercheur l'articulation indispensable entre l'étude de terrain en immersion participante, la mise à l'épreuve - par les traductions corporelles de ces théâtres dansés - de concepts et d'idées ethnocentristes, qu'ils soient de source indienne ou française. Nous avons également vu à quel point l'interdisciplinarité s'impose dans ce type de recherche: les outils méthodologiques puisés à la fois dans les champs historique, esthétique et anthropologique contribuent à générer une vision transculturelle de ces arts du spectacle vivant. Mais l'approche analytique repose sur l'abondance de descriptions extrêmement précises et érudites afin de démontrer les degrés de complexité d'un tel art, sur lesquels se fondent la créativité et la réinvention de son patrimoine. C'est pourquoi, pour pallier à un manque de publications sur le sujet et d'archives audiovisuelles et pour approfondir ces processus particuliers et esthétiques de patrimonialisation liés à la transmission orale et à ses éléments transculturels, il est important de structurer la méthodologie des jeunes chercheurs-artistes par trois éléments: une solide formation dans un style de théâtre dansé in situ en Inde, des entretiens avec les artistes locaux impliquant l'apprentissage de la langue et l'observation participante sur plusieurs terrains à la fois, à l'échelle locale, nationale et internationale. Car dans le champ des arts de la scène et du spectacle vivant, chercher rime à la fois avec préserver, réinventer et créer. 


\section{Notes}

${ }^{1}$ L'hégémonie encore actuelle de la première institution de Bharata-Nâtyam, Kalakshetra et de la formation qu'elle propose en langues tamoule et anglaise, occulte un grand nombre d'autres formes de patrimoine de ce style, notamment à Mysore. Cette ville d'un million d'habitants est située dans l'État du Karnataka, état voisin du Tamil Nadu. On y parle surtout le kannada. Notons que pour la première fois à Kalakshetra, en 2007, Leela Samson, nouvelle directrice de ce lieu, crée une mise en scène en langue kannada du texte de Purandaradasa, Dansaru Kanda Krishna (Samson, 2007, n.p.).

${ }^{2}$ En 1930, Anna Pavlova lui proposa en effet «[...] to see and learn real Traditional Indian Dance», phrase mentionnée dans l'ouvrage Rukmini Devi Arundale Birth Centenary Volume, Kalakshetra Foundation Publication. Notons que dans ses ouvrages en anglais et depuis les années 1970, Kapila Vatsyayan, l'une des grandes spécialistes indiennes du Bharata-Nâtyam et d'autres styles de théâtre dansé, utilise toujours les termes Indian Classical Dance.

${ }^{3}$ Comme c'est le cas pour ce temple, édifié d'abord au XII siècle puis au XVII siècle, les maharajas (pour l'orthographe voir les commentaires précédents) de Mysore ont fait de cette déesse leur emblème principal. Pour vaincre le démon Mahisasura menaçant de détruire l'univers, les trois plus grands dieux, Brahma, Vishnu et Shiva associent leurs pouvoirs pour créer une forme féminine brandissant dix bras armés. Il s'agit d'une forme de Durga et elle chevauche un lion pour combattre ses ennemis.

${ }^{4}$ Dans les années 1940 et 1960, selon le témoignage de H.R. Keshavamurthy, professeur de Bharata-Nâtyam formé par l'un des trois derniers maîtres du Palais, ces derniers enseignaient également aux danseurs de temples et accompagnaient avec eux des dizaines de processions. Ils se tenaient debout à dos d'éléphant, sur une plateforme en interprétant des poèmes dédiés à la déesse du temple, accompagnés par un orchestre assis sur un autre éléphant (Gaston, 1996, p.166).

${ }^{5}$ Selon K. Muralidhar Rao, le style de Tanjore se reconnaît encore actuellement chez les danseurs par les jatis, compositions rythmiques de danse pure (Narayana Rao, 1999, p.52-53).

${ }^{6}$ Ce qui est pérennisé encore aujourd'hui dans la majorité des répertoires de BharataNâtyam, ce sont deux pièces maîtresses de ce répertoire de Tanjore: le varnam et le tillana, qui comprennent principalement des textes en tamoul. Il est intéressant de noter que le tillana est un élément encore plus étranger au Bharata-Nâtyam que le varnam, puisqu’à l'origine il est la forme musicale (tarana) qui conclut les concerts de musique hindoustanie, donc du Nord de l'Inde. Cette pièce du récital a été introduite en Inde du Sud au XIX ${ }^{e}$ siècle, à la cour du Maharaja de Travancore puis elle a été intégrée dans les concerts de musique carnatique (Raghavendra Rao, 1998, p. 13-18).

${ }^{7}$ Fille d'un lutteur et d'une masseuse, tous deux employés au palais de Mysore, Jetti Tahamma a été nommée danseuse de cour en 1870, à l'âge de quinze ans. Elle a été contemporaine de Cinnaya Pillai, compositeur du palais de Tanjore invité à la cour de Mysore. Décédée en 1947, elle exerça son art jusqu'à l'âge de quatre-vingt-cinq ans sous le règne de Krishna Wadeyar IV (1902-1940). D'après l'étude de S. R. Bharatanatya dans $A$ Critical study, Mysore, publiée à Mysore en 1969, par la Sri Varalakshmi Academy of Fine Art, en 1969, elle contribua à développer dans les répertoires, des poèmes appelés çurnikai, 
composés notamment par le Maharaja Krishnaraja Wadeyar III (1811-1868). Après la conquête de l'État de Mysore par le Sultan musulman Tippu, puis sa chute en 1789, le règne de la famille royale hindoue des Wadeyar fut restauré. Elle encouragera un nouvel essor artistique des répertoires caractérisant sa tradition, en prenant exemple sur un modèle ancien de son histoire, celui du royaume Vijayanagar (1336-1565) et son mécénat des arts.

${ }^{8}$ Cette composition peut également s'adresser à la déesse de la scène (rangatidevata) ou à Bharatamuni, l'auteur présumé du Nâtya-Shâstra. Elle est précédée par un très court poème en vers non rythmé ou shloka du poète Kalidasa (extrait soit de Nataka Malavika Agnimitra, mis en musique dans le raga kalyani, soit de Hayagivam stuti, dans le nata raga). Outre ses danses transmises par Venkatalakhamma, nous avons également appris de K. Muralidhar Rao le çurnikaispatika patira, dédié à la déesse des arts Saraswati et mis en musique dans le raga arabhi.

${ }^{9}$ Il s'agit surtout de devaranama de Purandaradasa, de kriti de Mysore Sadashiva Ras ou de Vasudevacharya. Notons que ces textes dansés sont avant tout des compositions musicales qui font partie des récitals de chant, de flûte, de violon ou d'autres instruments.

${ }^{10}$ Il s'agit de deux vers extraits du poème d'introduction de Jayadeva (1991, n.p.).

${ }^{11}$ Sur son site internet Bhavara.com, cette artiste affirme préserver le Bharata-Nâtyam de Mysore en composant des danses sous forme de dramas, inspirés de Sri Purandhara Dasaru, de Punyakati et de Ramaharita.

${ }^{12}$ Lauréate du diplôme d'état Vidwat, titulaire d'un master en art de l'université de Mysore, cette artiste vit aux États-Unis, en Virginie, et a créé son école en 1994, Rishti School of Dance Training, dans laquelle elle transmet ce patrimoine de Mysore, tant en danse qu'en musique.

\section{Références}

BHABHA, Homi K. Les Lieux de la Culture. Traduction: Françoise Bouillot. Paris: Payot, 2007.

BHARUCHA, Rustom. The Politics of Cultural Practice. Londres: Athlone Press, 2000. CHARSLEY, Simon; KADEKAR, Laxmi Narayanan. Performers and their Arts. New Delhi: Routledge, 2006.

DEVI, Ragini. Dance Dialects of India. Delhi: Motilal Banarsidass, 1928.

GASTON, Anne-Marie. Bharata-Nâtyam: from temple to theatre. New Delhi: Manohar, 1996. JAYADEVA. Gita-Govinda. Traduction: Jean Varenne. Paris: Du Rocher/UNESCO, 1991. KAY, Ambrose. Classical Dances and Costumes of India. Londres: Adam/Charles Black, 1950.

KOTHARI, Sunil. New Directions in Indian Dance. Mumbai: Marg, 2000.

LÉGERET, Katia. La Gestuelle des Mains dans le Théâtre Dansé Indien. Paris: Geuthner, 2005. 
LÉGERET, Katia. Danse Contemporaine et Théâtre Indien: un nouvel art? Paris: PUV, 2010. NANDIKESHVARA, Abhinayadarpana. Traduction: P. S. R. Apparao. Hyderabad: Natyamala, 1997.

NARAYANA RAO, GuddehithluThimmappiah. With the Great Minds.Mangalore: Athree Book Centre, 1999.

RAGHAVENDRA RAO, K.Performing Arts: the focus of Mysore seminar. Sruti: la revue mensuelle de Chennai, Chennai, IBN, p. 13-18, nov. 1998.

RAO, Muralidhar Kasaragod. Nrityaloka. Mangalore: Athree Book Center, 1998.

ROSEMARY, Jeanes Antze. On Shifting Ground: continuity and change in the life of a Mysore court dancer. In: WATERHOUSE, David. Dance of India. Toronto: South AsianStudiesPapers, n. 10, 1998, n.p.

SATYANARAYANA, Y. Pushpanjali. Journal of the Madras Music Academy, Madras, The Music Academy Madras, v. XXXVII, n. I-IV, p. 98-105, 1966.

SAMSON, Leela. Leela Samson Speaks. The New Sunday Express, Chenai, 22 avr. 2007.

TARABOUT, Gilles. Passage à l'Art: l'adaptation d'un culte sud-indien au patronage artistique. Dans: ESCANDE, Yolande; SHAEFFER, Jean Marie. L'Esthétique: Europe, Chine et Ailleurs. Paris: You-Feng, 2003. P. 37-61.

VENKATARAMAN, Leela; PARASRICHA, Avinash. La Danse Classique Indienne: une tradition en transition. Paris: Lodi (EDL), 2003.

ZARRILLI, Phillip. When the Body Becomes all Eyes: paradigms, discourses and practices of power in Kalarippayattu, a South Indian martial art. New Delhi: Oxford University Press, 1998.

Katia Légeret est professeur en esthétique des arts de la scène au département théâtre de l'université Paris 8, directrice de l'équipe de recherche EA 1573 Scènes du monde, création, savoirs critiques. Katia Légeret alias Manochhaya mène également une carrière internationale dans le style Bharata-nâtyam, théâtre dansé de l'Inde. Elle a publié plusieurs ouvrages, dont Danse Contemporaine Indienne et Théâtre Indien: un nouvel art? (2010), Le Texte Étranger (2014) et Rodin et la Danse de Çiva (2014). E-mail: katia.legeret@univ-paris8.fr

Ce texte inédit, révisé par Annelyse Gayraud, est également publié en portugais dans ce numéro. 